\title{
Caracterización de Opus tesselatum procedentes de Ávila, España
}

\section{Geo-chemical Analysis of Opus tesselatum from Ávila, Spain}

María de los Reyes de Soto ${ }^{1}$, María Dolores Petit-Domínguez², Isabel Sonsoles de Soto ${ }^{3}$, Rosario García Giménez4, Isabel Rucandio ${ }^{5}$

Recibido 10/06/2014

Aceptado 01/09/2014

\section{Resumen}

Se han estudiado diversas muestras de Opus tesselatum procedentes de excavaciones arqueológicas de urgencia en Ávila (España) para determinar su origen y las tecnologías de producción utilizadas en su fabricación. Las teselas se han caracterizado por Espectrometría de Masas con fuente de Plasma de Acoplamiento Inductivo (ICP-MS), Difracción de Rayos X (XRD) y Espectrofotometría. Las muestras se corresponden con diferentes materiales con orígenes dispares (vidrios y rocas de varias áreas de aprovisionamiento). El color rojo en algunas teselas se debe principalmente a la presencia de cinabrio en su composición, lo que indica un movimiento comercial de materiales desde otras zonas de España ya que este mineral no está presente en las rocas de la provincia de Ávila.

Palabras clave: teselas, análisis mineralógico, análisis químico, Ávila, vidrio.

\begin{abstract}
Several Opus tesselatum samples from an emergency archaeological excavation in Ávila (Spain) have been studied in order to gather their origin and the production technologies used in their manufacture. Inductively coupled plasmamass spectrometry (ICP-MS), X-ray diffraction (XRD) and spectrophotometry were used to characterize the samples. The samples were made with different materials of different origin (glasses and rocks from different Spain areas). Red color in some Opus tesselatum samples is mainly due to cinnabar presence in their composition. It could be indicative of commercial movements between different Spanish sites, since this mineral is not present in rocks from Ávila.
\end{abstract}

Keywords: tessera, cinnabar, Ávila, glass.

\section{INTRODUCCIÓN}

Los mosaicos son una de las manifestaciones artísticas más importantes legada por los romanos tanto por su número como por su calidad y variedad (Blázquez Martínez, 1993). Hasta hace poco, los mosaicos han sido estudiados desde el punto de vista estilístico e iconográfico exclusivamente, si bien recientemente se han comenzado a emplear análisis arqueométricos con el fin de: (a) caracterizar los materiales (b) conocer las técnicas de fabricación para la obtención de sus componentes, las teselas, (c) establecer la ubicación de los centros de producción donde se fabricaban y (d) solventar los problemas para su conservación (Azzoni et alii, 2002; James, 2006).
1 Departamento de Prehistoria, Historia Antigua y Arqueología. Universidad de Salamanca, Salamanca.

2 Departamento de Química Analítica y Análisis Instrumental. Facultad de Ciencias. Universidad Autónoma de Madrid, Madrid.

3 Departamento de Ciencias del Medio Natural. Escuela Técnica Superior de Ingenieros Agrónomos. Universidad Pública de Navarra, Pamplona.
4 Departamento de Geología y Geoquímica. Facultad de Ciencias. Universidad Autónoma de Madrid, Madrid. rosario.garcia@uam.es

5 Centro de Investigaciones Energéticas, Medioambientales y Tecnológicas (CIEMAT). Madrid 
En los últimos años, se está incrementando el número de contribuciones arqueométricas dedicadas al estudio de los vidrios antiguos utilizados en la fabricación de estas teselas, sobre todo con respecto a su caracterización mineralógica y fisicoquímica, técnicas de producción, fuente de aprovisionamiento de materias primas y procesos de alteración de los mismos (Brill, 1999 y 2002; Foy et alii, 2003; Silvestri et alii, 2005; Silvestri, 2008; Foster y Jackson, 2010).

Los mosaicos de vidrio se consideran a menudo como la forma más excelente y elaborada de un mural así como la decoración espléndida de los pavimentos en la antigüedad, casi sustitutiva de los suelos marmóreos. Sin embargo, se tiene poco conocimiento acerca de cómo se manufacturaron los mosaicos y de la procedencia de sus materiales. Además, la tecnología de producción de mosaicos constituidos por vidrio coloreado era diferente y más compleja que la usada en la fabricación de vidrio transparente, debido a la necesidad de aditivos en la mezcla básica de colorantes y componentes opacificantes.

Algunos autores han abordado el problema de la clasificación de los mosaicos de vidrio a partir del análisis de sus componentes, identificando algunas diferencias importantes en base a sus características cronológicas y geográficas (Ruffini et alii, 1999;
Andreescu-Treadgold et alii, 2006; Vandini et alii, 2006). En algunos casos, por ejemplo en Gran Bretaña, esto ha ayudado a identificar la procedencia de los materiales empleados para la fabricación de mosaicos romanos (Neal y Cosh, 2002 y 2009; Allen et alii, 2007; Young et alii, 2008; Hayward, 2009), en otros casos, aparecen discrepancias entre lo que se infiere del análisis de sus componentes y la procedencia local de las teselas sugerida por evidencias históricas.

El objetivo principal de este trabajo es caracterizar las teselas encontradas en dos mosaicos de la ciudad de Ávila y demostrar la posible relación con el área de aprovisionamiento de materiales cercana. Así como conocer las técnicas de fabricación para la obtención de sus componentes.

\section{2. ÁREA DE ESTUDIO Y DESCRIPCIÓN DE LOS YACIMIENTOS}

\section{1. Área de estudio}

El área de estudio se desarrolla en dos solares de la ciudad de Ávila, la necrópolis de la Iglesia de San Pedro, en la Plaza del Ejército y el antiguo Hotel Continental, en la Plaza de la Catedral (Figura 1).

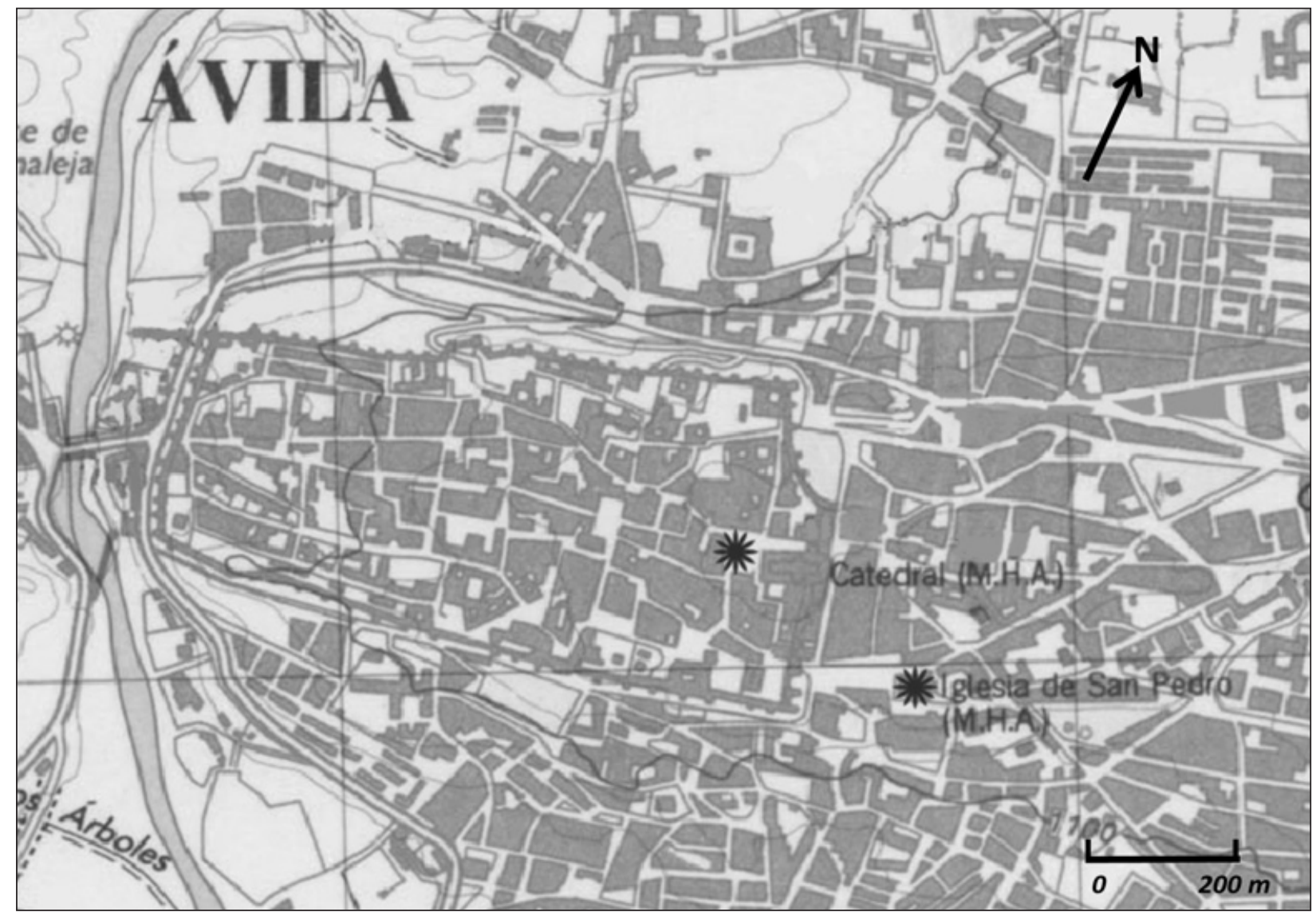

Figura 1. Plano de la ciudad de Ávila con los yacimientos señalados estudiados (Modificado del Plano Topográfico Nacional de España) 
Por paradójico que pueda resultar se posee un conocimiento realmente exiguo de la época romana en la provincia de Ávila, ya que son limitadas las fuentes escritas junto a los restos arqueológicos (Mariné Isidro, 1998). No obstante, en los últimos años este vacío se ha ido diluyendo gracias, en gran medida, a las excavaciones de urgencia obligatorias que se realizan en trabajos de construcción.

La ciudad de Ávila se localiza en la Meseta Norte, en la Comunidad Autónoma de Castilla y León, a 1131 metros de altitud, sobre una loma que cierra el valle Amblés. Posee un poblamiento romano atestiguado, por lo menos, desde el siglo I a.C. (Quintana López et alii, 2003-2004), sin embargo, el origen romano de la ciudad es uno de los temas más debatidos por los expertos. De este modo, Rodríguez Almeida, uno de los mayores especialistas del Ávila romana, o María Mariné, consideran que se trata de un campamento romano estable, cuyo cardo y decumano se cruzarían en la actual plaza mayor abulense (plaza de la Victoria o plaza del Mercado Chico). Esta estructura campamental sería respetada en época medieval, así como algunas zonas de la muralla actual, donde se conservarían restos de la anterior posible muralla romana, como la puerta de San Vicente, donde se ha detectado, en la actualidad, una torre de planta cuadrada de origen romano (Mariné Isidro, 1998; Rodríguez Almeida, 2003). Existen más teorías sobre el origen de la ciudad, así Barraca de Ramos se decanta por un esquema repoblador, teniendo la muralla un esquema medieval, no romano (Barraca de Ramos, 1999). Mangas Manjarrés se inclina por un establecimiento romano administrativo, desde el que podría mantenerse el orden de las zonas cercanas (Mangas Manjarrés, 1996). Hernando Sobrino considera que el plano urbanístico puede corresponder a un origen campamental romano, ya que algunas ciudades del Bajo Imperio fueron planificadas al modo militar, pero no por ello deben ser campamentos, por lo que en realidad se inclina hacía que la fundación de Ávila mantuvo el sistema viario (Hernando Sobrino, 2005), y por último, algunos de los últimos investigadores en pronunciarse sobre este tema fueron Quintana, Centeno y Ruíz, quienes gracias a los datos compilados en las excavaciones de la Plaza del Mercado Grande, consideran que se trata de un establecimiento indígena del siglo I a.C., poco romanizado hasta que en el siglo I d.C. se romaniza con mayor fuerza (Centeno Cea et alii, 2003).

Al considerar la Antigüedad Tardía, época a la que corresponden las muestras analizadas, se ha señalado como característica principal de este periodo, su desconocimiento (Barraca de Ramos, 2012), aunque las recientes actuaciones arqueológicas desarrolladas han permitido obtener nuevos datos de esta etapa histórica. Se han conseguido algunos hallazgos aislados, como restos de fragmentos cerámicos, asolados de muros y materiales procedentes de necrópolis (Barraca de
Ramos, 2012), incluso se han encontrado muros de época tardoantigua tanto dentro como fuera del perímetro amurallado, caso de la excavación de Santa María la Antigua, el Palacio de los Velada o la Catedral.

En general, la cultura material documentada de época tardoantigua es bastante pobre y mucho más la relacionada con restos de mosaicos o teselas. Únicamente han aparecido teselas en la calle de la Muerte y la Vida (Fabián García, 2007), en la iglesia de La Antigua (Barraca de Ramos, 1993) y con el presente trabajo en la necrópolis de San Pedro y en el Hotel Continental. Por el contrario, en el mundo rural abulense sí que se conocen algunas villas que estuvieron decoradas con mosaicos, como "Las Claveranas" (Mancera de Arriba) (Mariné Isidro, 1984), "El Vergel" (San Pedro del Arroyo) (Foramen, 2012) o la ermita de Santa María de la Vega (Piedrahita) (Mariné Isidro, 1998), pero todas ellas vinculadas con asentamientos rurales y nunca en un contexto urbano.

\subsection{Descripción de los yacimientos}

La necrópolis de la iglesia de San Pedro fue descubierta en 1959 gracias a los trabajos de pavimentación de la zona, por lo que se decidió realizar un estudio y documentación de la misma. Los primeros resultados indicaron que Santa María y San Pedro tenían estructuras que se podían estudiar desde el punto de vista arqueológico, y se emprendió un análisis separado de las mismas, pero siempre teniendo en cuenta su fuerte vinculación (Barraca de Ramos, 1993).

En el sector de Santa María la Antigua se documentaron distintas producciones cerámicas, algunos estucos de época tardorromana, un mosaico totalmente fragmentado y varias teselas de distintos colores (Barraca de Ramos, 1993). La zona del circuito de San Pedro debió tener cierta importancia ya desde época romana que tiene corroborada la existencia de establecimientos habitacionales superpuestos (Barraca de Ramos, 1993). Sobre los restos de época tardoantigua, con los datos disponibles, no se puede saber su funcionalidad, aunque, según la investigadora mencionada, podría ser de tipo religiosa (Barraca de Ramos, 1993).

Las muestras que se analizan en el caso actual proceden del sector oriental del yacimiento, próximo a la cabecera del templo. Las teselas recogidas en la necrópolis de San Pedro se encontraban descontextualizadas, pero concentradas en los cimientos de una construcción funeraria de los siglos IV-V, por lo que podrían formar parte de un monumento funerario o de la decoración de estas estructuras.

En el Hotel Continental los datos de los que se dispone son realmente escasos. Se trata de uno de los pocos ejemplos de mosaicos romanos en la ciudad de Ávila, sino el único, vinculados a una estructura doméstica arrasada hasta el nivel de los mosaicos de 
época tardorromana. Con posterioridad, se construyó una estructura de época visigoda, sobre la que más tarde se hicieron unos fosos de época plenomedieval, una casona en época moderna y por último, en el siglo XIX, el hotel actual, por lo que el estado de conservación no es bueno. Las muestras, en este caso, formaban parte de los pavimentos de una habitación del siglo IV, consistente en un mosaico geométrico, en gran parte destruido por las construcciones posteriores de la Edad Media (Figura 2).
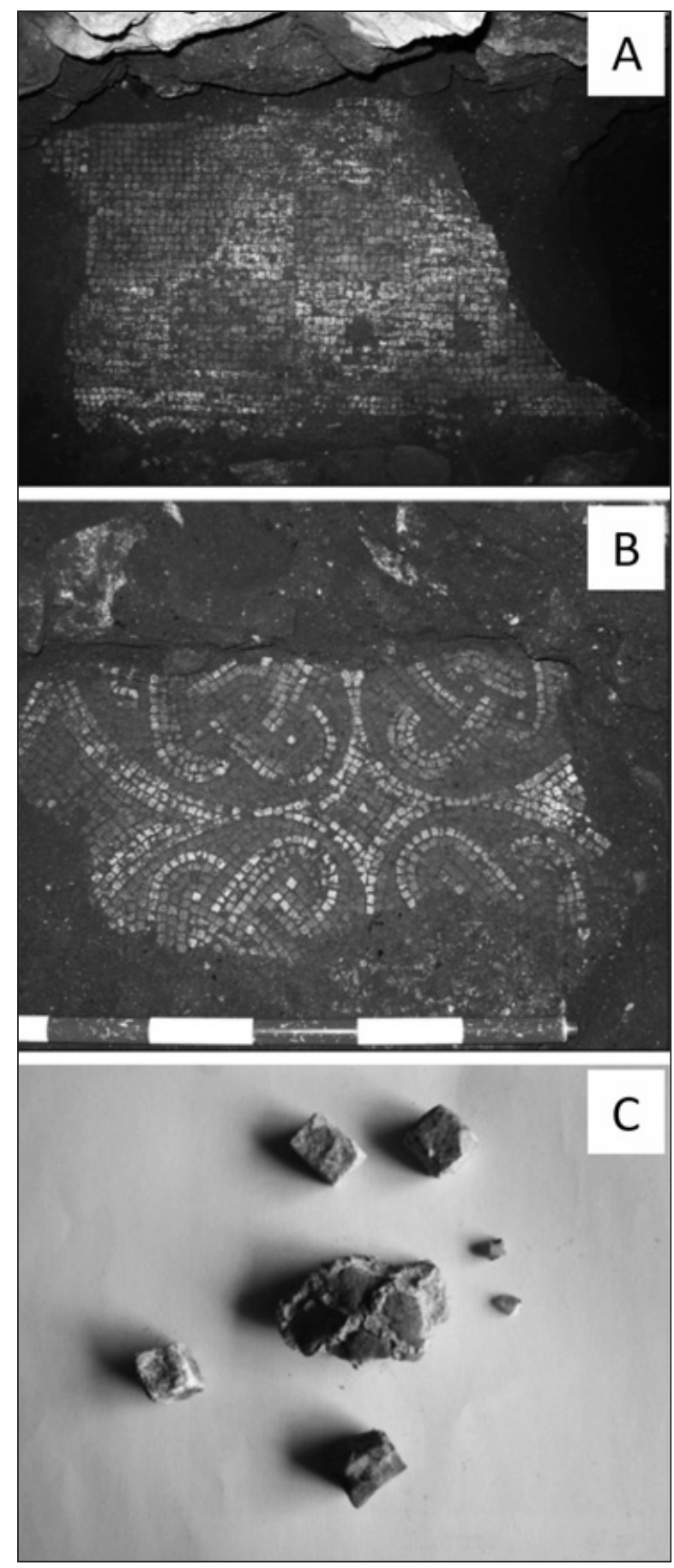

Figura 2. Ejemplos de mosaicos (A y B) y teselas (C)

\section{MATERIALES Y MÉTODOS}

\subsection{Materiales}

En este trabajo se presenta la caracterización arqueométrica de ocho teselas extraídas de diferentes mosaicos procedentes de las excavaciones de urgencia realizadas. Son muestras representativas de las teselas de diferentes colores encontradas en las excavaciones mencionadas. A menudo tienen una estructura irregular, muestran fracturas concoides, sobre todo las de color azul y crema. Además se han seleccionado otras de color rojo, verde y blanco, con forma de prisma rectangular y dimensiones medias de $1 \mathrm{~cm}$ x $1 \mathrm{~cm}$ x $1,5 \mathrm{~cm}$. Las correspondientes a la necrópolis de la parroquia de San Pedro, se las clasifica como: Verde, Crema, Azul y Rojo 1 y las relativas a la excavación del antiguo Hotel Continental, son las denominadas: Rojo 2, Blanco 1, Blanco 2 y Blanco 3.

\subsection{Métodos}

Se han analizado ocho teselas por difracción de rayos X (XRD) y Espectrometría de Masas con fuente de Plasma de Acoplamiento Inductivo (ICP-MS). El objetivo es el estudio de las materias primas y la tecnología de producción para reunir información sobre la presencia y distribución de las fases cristalinas y sobre el entorno químico de los elementos cromóforos.

\subsubsection{Color de las teselas}

Se utilizó un espectrofotómetro Minolta CM2500d, con procesador de datos. Para la realización de las medidas, según normativa, se seleccionó un iluminante D65 y observador estándar 10 grados, en un espacio de color CIELAB, estandarizado internacionalmente. Este espacio de color consiste en un sistema tridimensional compuesto por tres ejes: L* (eje correspondiente a la luminosidad, transición blanco-negro); a* (eje transición rojo-verde); b* (eje transición amarillo-azul), que constituyen las denominadas coordenadas CIE para la determinación del color.

\subsubsection{Análisis Mineralógico}

El análisis mineralógico se llevó a cabo por XRD usando el método de polvo en muestra total (Moore y Reynolds, 1997), utilizando un difractómetro SIEMENS D-5000 con ánodo de $\mathrm{Cu}$ trabajando a $30 \mathrm{~mA}$ y $40 \mathrm{kV}$ con rendijas de divergencia y recepción de 2 y $0,6 \mathrm{~mm}$, respectivamente. Se han semicuantificado los minerales cristalinos identificados según Brindley y Brown (1980), aplicando los poderes reflectantes de Schultz (1964) y Barahona (1974). 


\subsubsection{Análisis químico}

La disolución de las muestras se llevó a cabo de la siguiente manera: una cantidad mínima de muestra se trató con ácido fluorhídrico en un recipiente abierto de teflón y se calentó en placa calefactora. A continuación se adicionó agua regia y se calentó de nuevo hasta sequedad. El residuo se disolvió con 1 $\mathrm{ml}$ de ácido clorhídrico concentrado diluyendo posteriormente con agua en matraces volumétricos de teflón. Se utilizó agua ultrapura en todo el proceso y los reactivos utilizados fueron de grado analítico. Se analizaron 68 elementos por ICP-MS, en un espectrómetro Perkin-Elmer 6000 Elan Sciex equipado con un muestreador automático COMO 91. Los elementos mayoritarios analizados son: $\mathrm{Al}, \mathrm{Ca}, \mathrm{Fe}, \mathrm{K}$, $\mathrm{Mg}, \mathrm{Mn}, \mathrm{Na}, \mathrm{P}$ y Ti determinándose Si por diferencia; y los elementos traza: Ag, As, Au, B, Ba, Be, Bi, $\mathrm{Ce}, \mathrm{Cd}, \mathrm{Co}, \mathrm{Cs}, \mathrm{Cu}, \mathrm{Cr}$, Dy, Er, Eu, Ga, Gd, Ge, Hf, $\mathrm{Hg}$, Ho, In, Ir, La, Li, Lu, Mo, Ni, Nb, Nd, Os, Pb, $\mathrm{Pd}, \mathrm{Pr}, \mathrm{Pt}, \mathrm{Re}, \mathrm{Rb}, \mathrm{Rh}, \mathrm{Ru}, \mathrm{Sb}, \mathrm{Sc}, \mathrm{Se}, \mathrm{Sm}, \mathrm{Sn}, \mathrm{Sr}, \mathrm{Ta}$, $\mathrm{Tb}, \mathrm{Te}, \mathrm{Th}, \mathrm{Tl}, \mathrm{Tm}, \mathrm{V}, \mathrm{W}, \mathrm{Y}, \mathrm{Yb}, \mathrm{Zn}$ y Zr. Simultáneamente, se analizaron muestras en blanco, muestras patrón y muestras duplicadas como control de calidad. Además, para evaluar la exactitud del análisis, se utilizaron varios materiales de vidrio de referencia certificados: soda-lime flat glass NIST SRM 620, soda-lime float glass NIST SRM 1830 y soft borosilicate glass NIST SRM 1411. Los errores obtenidos para todos los elementos con respecto a los valores certificados fueron inferiores al $5 \%$ en todos los casos.

\subsubsection{Análisis estadístico}

Los resultados obtenidos se sometieron a un procesamiento estadístico de los datos mediante el programa Statgraphics Plus 5.0 para Windows ${ }^{\circledR}$. Se ha realizado un estudio estadístico multivariante, mediante análisis de componentes principales, basado en la composición química de elementos traza. Esta herramienta estadística se utiliza para clasificar un conjunto de datos tratando de establecer las posi- bles conexiones entre las diferentes muestras y los elementos químicos analizados.

\section{RESULTADOS Y DISCUSIÓN}

\subsection{Determinación de color}

El color de las teselas se midió con el fin de evitar una descripción subjetiva de las muestras. Las coordenadas de color de las muestras se presentan en la Figura 3. Todas son L positivo. Las teselas blancas tienen las mismas coordenadas con un parámetro a negativo y b positivo. Las rojas presentan los parámetros a y b positivos. La tesela verde es negativa para a y positiva para b. La azul y la crema presentan las coordenadas a y b con el mismo signo.

4.2. Evaluación de la composición mineralógica mediante Difracción de Rayos X (XRD).

El estudio mediante difracción de rayos $\mathrm{X}$ confirma que las teselas de tonos azul y crema, localizadas en San Pedro, fueron elaboradas con material vítreo. Por el contrario las teselas de tonos verde y rojo (rojo 1) de San Pedro y todas las encontradas en la Plaza de la Catedral (blanco 1, 2 y 3 y rojo 2) se realizaron a partir de materal pétreo. Se puede considerar como teselas de abundante fase vítrea las correspondientes a los colores azul y crema (Figura 4A), ambas con una amplia banda entre 15 y $20^{\circ} 2 \theta$ En el difractogarma de la tesela azul se identifica cuarzo y arcillas de tipo micáceo y coalinítico, con una banda en torno a 3,00 identificada como calcita (Figura 4A). Por su parte la tesela de color crema también denota la existencia de

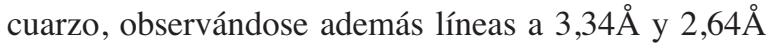
que corresponderían al óxido de estaño (casiterita) que incluso coincidirían con la bindheimita, amarillo de Nápoles o $\mathrm{Pb}_{2} \mathrm{Sb}_{2} \mathrm{O}_{7}$. Este tipo de vidrio, podría ser transportado por los vidrieros o fabricado in situ por los mismos.

Los difractogramas de las teselas de tonos rojo, blanco o verde (Figura 4B) muestran un fondo plano, que indica menor contenido en fase vítrea. Entre las

\begin{tabular}{|llccc|}
\hline UBICACIÓN & COLOR & L* & $\mathbf{a}^{*}$ & $\mathbf{b}^{*}$ \\
\hline San Pedro & Verde & 50.03 & -9.5 & 8.12 \\
& Crema & 45.89 & 0.69 & 12.65 \\
& Azul & 44.89 & -0.35 & -12.67 \\
& Rojo1 & 46.42 & 23.06 & 1.8 \\
\multirow{5}{*}{ Plaza Catedral } & Rojo2 & 43.56 & 14.94 & 7.9 \\
& Blanco1 & 52.29 & -2.26 & 9.93 \\
& Blanco2 & 52.48 & -1.88 & 12.15 \\
& Blanco3 & 59.93 & -1.45 & 6.20 \\
\hline
\end{tabular}

Figura 3. Coordenadas de color de las teselas según coordenadas CIE (Comisión Internacional de l’Eclairage) 

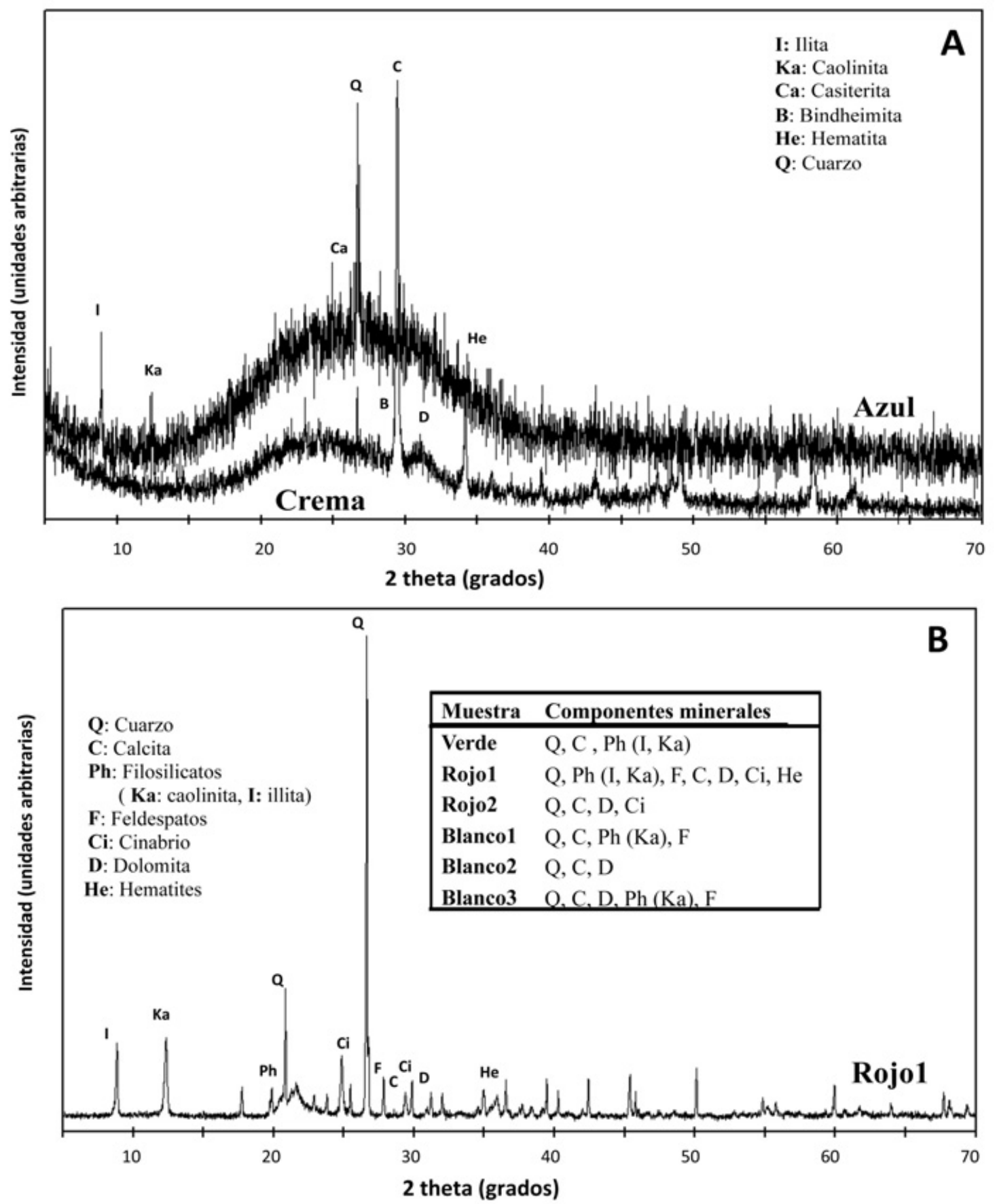

Figura 4. DRX de teselas. A. con abundante fase amorfa. B. cristalinas

fases cristalinas identificadas destaca el alto contenido en filosilicatos (caolinita e ilita) y en cuarzo, con pequeñas cantidades de feldespatos, calcita y dolomita. En la Figura 4B se recoge una tabla resumen de los resultados para los distintos componentes de estas muestras. El difractograma representado en esta misma figura, corresponde a la muestra Rojo1 que contiene cinabrio, cuarzo, feldespatos, hematita y filosilicatos. El color rojo procede de la potencia- ción de la tonalidad debida a la presencia de cinabrio y de hematita en su composición y así lo ha mencionado Duran (2011) en muestras en las que coexisten cinabrio con cantidades elevadas de óxido de hierro. En España hay dos zonas que geológicamente se caracterizan por la presencia de este mineral; el área de Riaño (Norte de España) (Hunt Ortiz, 2003) y el distrito minero de Almadén (Sureste de España) (Fernández Ochoa et alii, 2002). Sin embargo, este 
mineral no está presente en las rocas de la provincia de Ávila, por lo tanto, la presencia de teselas que contienen cinabrio indica que estos materiales fueron transportados hasta la ciudad desde Riaño (Norte de España) (Hunt Ortíz, 2003) o desde Almadén (Sureste de España) (Álvarez Martín, 1983, Fernández Ochoa et alii, 2002). El análisis químico de las muestras y su posterior comparación con ejemplares de estos dos distritos mineros permitirá resolver la cuestión del origen de las teselas rojas encontradas en la ciudad de Ávila.

En las teselas blancas se han identificado cuarzo y calcita, así como feldespato, filosilicatos, y dolomita en algunas de ellas. Teselas con esta composición mineralógica suelen estar formadas por calizas micríticas (Morillo y Salido, 2011-12). En el ámbito próximo a la ciudad de Ávila aparecen rocas compuestas por los minerales descritos (calizas con una proporción del 10\% de cuarzo y feldespato y areniscas arcósicas con cemento carbonatado), con lo que podría asociarse el origen de este tipo de teselas con las proximidades del entorno. Sin embargo, la ausencia de fósiles en las muestras hace muy difícil afirmar esta asociación. Un análisis micropaleontológico podría resolver esta cuestión pero las teselas blan- cas de estos yacimientos arqueológicos no poseen microfósiles.

Por último, las teselas de color verde, de composición mineralógica consistente en cuarzo, calcita y filosilicatos (clorita), se pueden asociar petrológicamente, dada su alto contenido en clorita, con esquistos verdes de los que aparecen en el entorno de Ávila.

\subsection{Composición química}

Los resultados de los componentes químicos mayoritarios de las teselas se resumen en forma gráfica en la Figura 5 y los de los componentes químicos minoritarios se recogen en la Figura 6.

La Figura 5 consta de cuatro gráficas con distintas escalas en las que se distribuyen los elementos mayoritarios. En general, las muestras se componen esencialmente de $\mathrm{SiO}_{2}, \mathrm{CaO}$ y $\mathrm{Na}_{2} \mathrm{O}$. Estos dos últimos óxidos, junto con los correspondientes de $\mathrm{Mg}$ y $\mathrm{K}$, son los esenciales incorporando, al mismo tiempo, cantidades significativas de elementos de metales de transición ( $\mathrm{Co}, \mathrm{Cu}, \mathrm{Fe}, \mathrm{Ni}, \mathrm{Mn}$, etc.) que normalmente tienden a entrar en las redes cristalinas como soluciones o formar dispersiones coloidales que proporcionan el color del vidrio (elementos cromóforos).

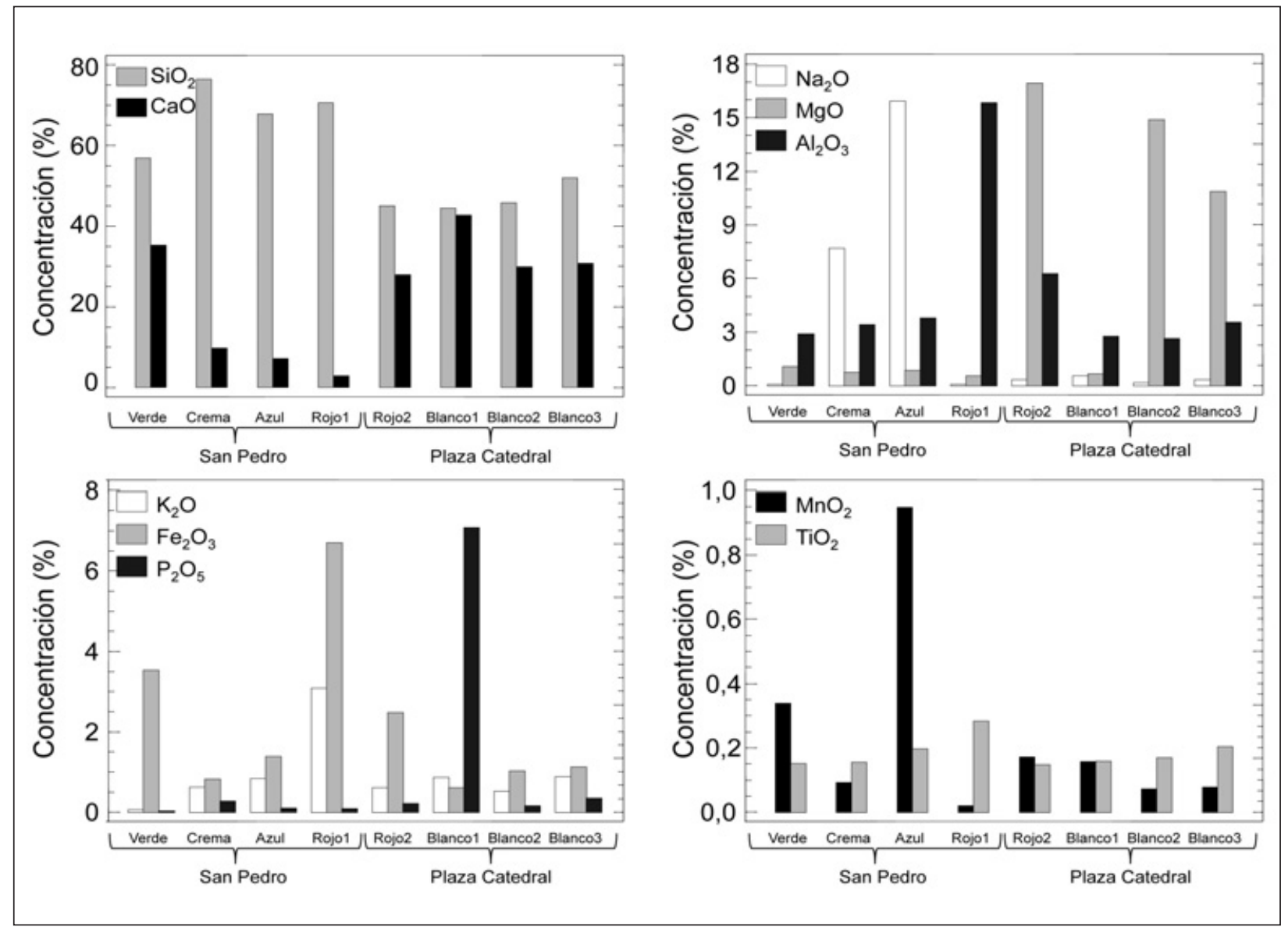

Figura 5. Resultados de los mayores constituyentes para todas las muestras. 


\begin{tabular}{|c|c|c|c|c|c|c|c|c|}
\hline & \multicolumn{4}{|c|}{ SAN PEDRO } & \multicolumn{4}{|c|}{ PLAZA CATEDRAL } \\
\hline & Verde & Crema & Azul & Rojo1 & $\underline{\text { Rojo2 }}$ & Blanco1 & Blanco2 & $\underline{\text { Blanco3 }}$ \\
\hline $\mathbf{B a}$ & 26 & 198 & 413 & 764 & 154 & 593 & 89 & 176 \\
\hline $\mathrm{Ce}$ & 1 & 15 & 9 & 39 & 35 & 10 & 17 & 17 \\
\hline Co & 8 & 3 & 22 & 2 & 3 & 3 & 3 & 4 \\
\hline $\mathrm{Cu}$ & 402 & 517 & 10745 & 251 & 1203 & 519 & 296 & 298 \\
\hline Hg & 0 & 0 & 1 & 226 & 28 & 4 & 0 & 1 \\
\hline La & 1 & 9 & 4 & 20 & 18 & 4 & 7 & 7 \\
\hline $\mathbf{L i}$ & 26 & 3 & 7 & 18 & 7 & 16 & 13 & 16 \\
\hline Nd & 5 & 8 & 6 & 22 & 13 & 6 & 9 & 8 \\
\hline $\mathrm{Ni}$ & 63 & 9 & 31 & 14 & 20 & 11 & 10 & 10 \\
\hline $\mathbf{P b}$ & 40 & 44546 & 1755 & 49 & 425 & 383 & 114 & 102 \\
\hline $\mathbf{R b}$ & 8 & 21 & 23 & 104 & 327 & 38 & 27 & 45 \\
\hline Sb & 1 & 2557 & 1456 & 6 & 40 & 32 & 28 & 5 \\
\hline Sn & 17 & 275 & 530 & 15 & 60 & 29 & 17 & 18 \\
\hline $\mathrm{Sr}$ & 272 & 373 & 460 & 82 & 0 & 534 & 130 & 203 \\
\hline $\mathbf{U}$ & 0 & 8 & 4 & 0 & 4 & 0 & 4 & 6 \\
\hline w & 2 & 3 & 20 & 6 & 2 & 0 & 1 & 3 \\
\hline $\mathbf{Y}$ & 21 & 8 & 10 & 14 & 23 & 9 & 6 & 8 \\
\hline $\mathrm{Zn}$ & 83 & 85 & 344 & 51 & 342 & 260 & 45 & 73 \\
\hline $\mathrm{Zr}$ & 2 & 64 & 69 & 68 & 10 & 19 & 15 & 29 \\
\hline
\end{tabular}

Figura 6. Resultados (expresados en ppm)

de los menores constituyentes y elementos traza para todas las muestras.

La sílice, $\mathrm{SiO}_{2}$, alcanza valores superiores al $45 \%$ en todas las teselas. La cal, $\mathrm{CaO}$, está presente en cantidades importantes (27-43\%) excepto en las muestras Crema, Azul y Rojo 1 en las que el contenido varía entre 2 y $10 \%$. El $\mathrm{Na}_{2} \mathrm{O}$ aparece de forma destacada en la muestra Azul (16\%) lo que hace que, junto con la composición que presenta en otros componentes mayoritarios, podría considerarse como perteneciente a los vidrios Romanos típicos de la época Imperial (Arletti y col, 2006). El $\mathrm{Na}_{2} \mathrm{O}$ en la muestra Crema tiene también un alto contenido $(7,7 \%)$ mientras que en el resto de los ejemplares se alcanzan contenidos inferiores al 0,6\%. En el caso del $\mathrm{MgO}$, las muestras Rojo 2, Blanco 2 y Blanco 3 son llamativas por sus concentraciones, comprendidas entre 10 y $17 \%$, y en la muestra Rojo1 es importante su alto contenido en $\mathrm{K}_{2} \mathrm{O}(3 \%)$ mientras que en la Verde su bajo valor $(0,052 \%)$.

Hay otro grupo de elementos, cuya concentración puede expresarse en óxidos y están representados en la Figura $5\left(\mathrm{Al}_{2} \mathrm{O}_{3}, \mathrm{Fe}_{2} \mathrm{O}_{3}, \mathrm{P}_{2} \mathrm{O}_{5}, \mathrm{MnO}_{2}\right.$ y $\left.\mathrm{TiO}_{2}\right)$ que según su contenido en las muestras se pueden considerar mayoritarios o minoritarios. En este sentido, también hay un conjunto de elementos que se consideran minoritarios, los cuales fueron añadidos intencionadamente en algunos ejemplares al objeto de potenciar alguna propiedad del material y que se encuentran resumidos en la Figura 6 expresados como elementos ( $\mathrm{Cu}, \mathrm{Pb}$ y $\mathrm{Sb})$. En esta misma figura también se aportan los resultados de los elementos considerados como traza $(<1000 \mathrm{ppm})$ que suelen provenir de impurezas incluidas en los materiales empleados para la fabricación de las teselas.

En la Figura 7 se representa el análisis mediante componentes principales obtenido con los datos de los elementos recogidos en la Figura 6. El propósito de este análisis es lograr un pequeño número de combinaciones lineales de las 19 variables (Ba, Ce, Co, $\mathrm{Cu}, \mathrm{Hg}, \mathrm{La}, \mathrm{Li}, \mathrm{Nd}, \mathrm{Ni}, \mathrm{Pb}, \mathrm{Rb}, \mathrm{Sb}, \mathrm{Sn}, \mathrm{Sr}, \mathrm{U}, \mathrm{W}, \mathrm{Y}$, $\mathrm{Zn}$ y $\mathrm{Zr}$ ) que explican la mayoría de la variabilidad en los datos. Este procedimiento es útil para clasificar los datos en grupos que comparten una composición química similar. En este caso, se han extraído cinco componentes que unidas explican el $94,7 \%$ de la variabilidad en los datos originales. De ellas se han representado gráficamente la componente 1 frente a la 2, ya que son las componentes que reflejan el mayor porcentaje de varianza $(33,8 \%$ y $23,9 \%$, respectivamente). Como se puede observar en esta figura, las muestras se encuentran perfectamente distribuidas según su color. Así, en el cuadrante superior izquierdo se detecta la muestra Verde, con importantes contenidos de $\mathrm{Ni} \mathrm{y} \mathrm{Cu}$, que junto con el $\mathrm{Fe}_{2} \mathrm{O}_{3}$ (aproximadamente $3.5 \%$ ) le confieren el color a la tesela. También es destacable en esta muestra el alto contenido en $\mathrm{Sr}$ (272 ppm). 


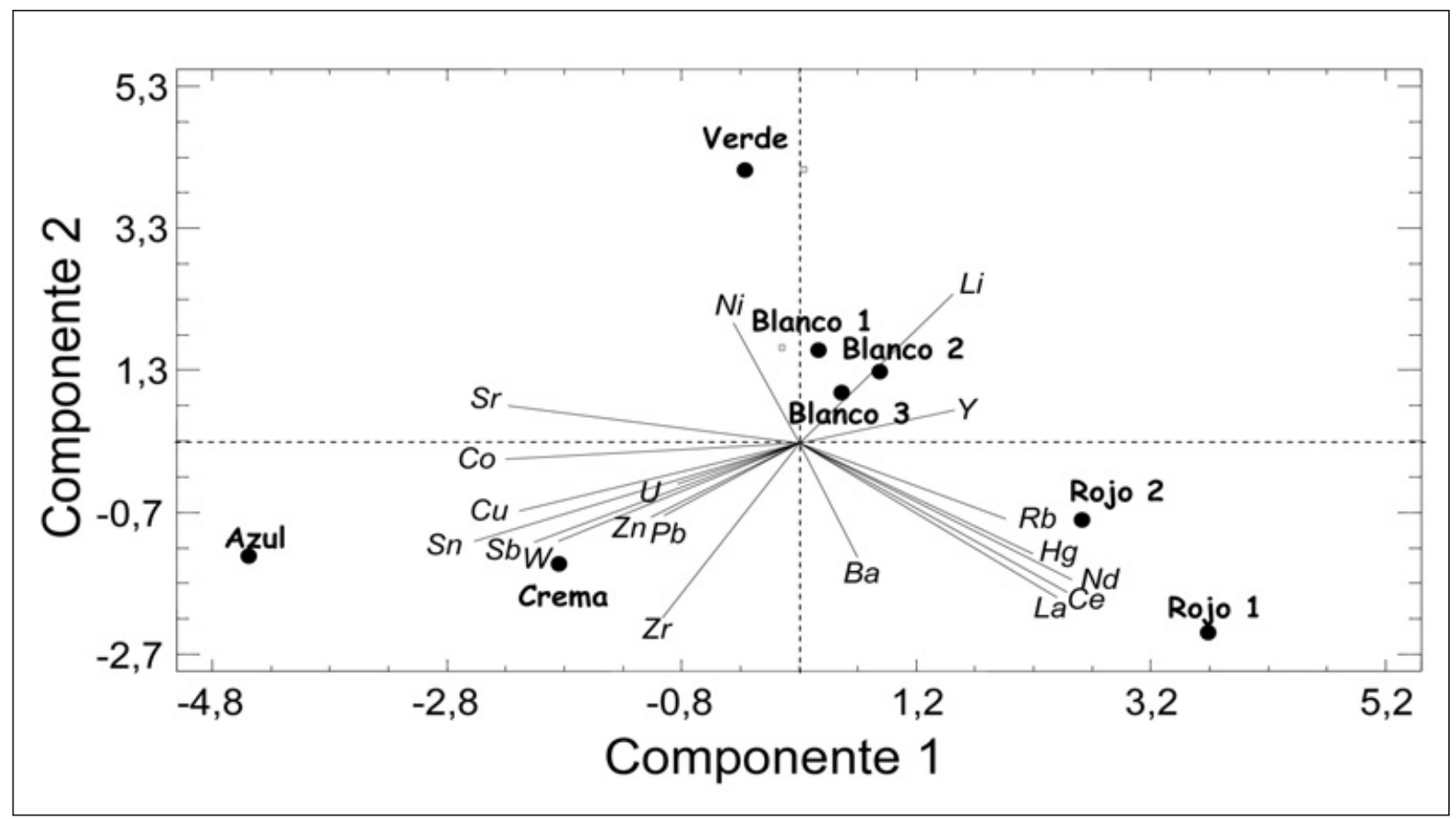

Figura 7. Análisis de componentes principales

Bajando al cuadrante inferior izquierdo aparecen las teselas Azul y Crema. El vidrio Azul debe su coloración a la alta concentración de $\mathrm{Cu}$ (cercano al 1\%), junto con contenidos importantes de Co (22 ppm). La presencia de $\mathrm{Cu}$ puede estar también correlacionada con el uso de vidrio reciclado en la mezcla (Silvestri et alii, 2006). El Co aparece asociado con Al, Fe, Mn y Zn (Jackson, 2005; Shortland et alii, 2007). La alta concentración de $\mathrm{MnO}_{2}$ (casi 1\%) se vincula con la fabricación del vidrio azul a base de cenizas de plantas (Degryse y Schneider, 2008). La tesela Azul junto con la Crema tienen una elevada concentración de $\mathrm{Sb}$ (1456 y 2557 ppm, respectivamente), $\mathrm{Pb}$ (1755 ppm y casi $4.5 \%$, respectivamente) y $\mathrm{Sn}(530$ y $275 \mathrm{ppm})$.

En el cuadrante inferior derecho se localizan agrupadas las dos muestras rojas. Ambas con una composición bastante diferente si consideramos los mayores constituyentes: la muestra Rojo1 presenta la mayor concentración de $\mathrm{Al}_{2} \mathrm{O}_{3}$ (casi $16 \%$ ), $\mathrm{Fe}_{2} \mathrm{O}_{3}$ (próxima a $7 \%$ ) y $\mathrm{K}_{2} \mathrm{O}$ (más de $3 \%$ ), mientras que la muestra Rojo 2 es la de mayor contenido en $\mathrm{MgO}$ (16-17\%) identificado por XRD como dolomita. En cuanto a los elementos traza, en ambas destaca la presencia de $\mathrm{Hg}$, circunstancia que se ha asociado al mineral cinabrio.

Por último, en el cuadrante superior derecho, y muy cerca del origen de coordenadas, se encuentran las tres muestras blancas con elevadas concentraciones de $\mathrm{CaO}$ que se relacionan con la presencia de calcita y la presencia de $\mathrm{Sr}$ y $\mathrm{Pb}$, ambos elementos relacionados geoquímicamente con la aparición de carbonatos, estroncianita y cerusita, paragenéticos con la calcita.

\section{CONCLUSIONES}

Las tesserae de los mosaicos estudiados en Ávila mezclan materiales de distinta naturaleza y procedencia (vidrios y rocas de diferentes orígenes y composición mineralógica)

El estudio mediante difracción de rayos X confirma que las teselas de tonos azul y crema, localizadas en San Pedro, fueron elaboradas con material vítreo. Por el contrario las teselas de tonos verde y rojo (rojo 1) de San Pedro y todas las encontradas en la Plaza de la Catedral (blanco 1, 2 y 3 y rojo 2) se realizaron a partir de material pétreo.

Las muestras rojas contienen mercurio por la existencia de cinabrio en su composición mineralógica. Dado que las rocas existentes en las cercanías de la ciudad de Ávila no contiene cinabrio, la presencia de este mineral indica una vez más el tráfico comercial. Además, estas teselas rojas localizadas en dos zonas diferentes de la ciudad de Ávila, todas con cinabrio, hace pensar bien en la reutilización de un mosaico anterior a otro más tardío o bien, al ser de la misma época, siglo IV, en una misma etapa constructiva, quizá trabajadas por el mismo artista, aunque de composición diferente.

Las muestras de color azul corresponden con un vidrio azul de alta concentración en cobre lo que le atribuye un carácter reciclado. También presenta concentraciones importantes de Co, lo que a su vez está asociado con contenidos destacados de Al, Fe, Mn y $\mathrm{Zn}$. La existencia de Mn indica fabricación del vidrio azul a base de cenizas de plantas. 
Las muestras blancas se corresponden con carbonatos que pueden haber sido transportadas de yacimientos próximos a la ciudad. Lo mismo sucede con las teselas verdes que son rocas a base de cuarzo, calcita y clorita, asociadas, posiblemente, a los esquistos verdes que aparecen en el entorno de Ávila.

\section{Agradecimientos}

Agradecemos a los arqueólogos de Castellum Sociedad Cooperativa de Ávila, su gentileza en la aportación de materiales y datos cronológicos de los mismos.

\section{Bibliografía}

Allen, J.R.L., Fulford, M.G., Todd, J. (2007): Burnt Kimmeridgian shale at Early Roman Silchester, south-east England, and the Roman Poole-Purbeck complex-agglomerated Geomaterials Industry. Oxford. Journal of Archaeology 26, 167 - 191.

Álvarez Martín, J.B. (1983): Estudio sedimentológico, geoquímico y metalogénico en las capas de San Nicolás y San Pedro (Mina de Almadén). Tesis Doctoral. Universidad Autónoma, Madrid, 285 pp.

Andreescu-Treadgold, I., Henderson, J., Roe, M. (2006): Glass from the mosaics on the west wall of Torcello Basilica. Arte Medievale V, 2-140.

Arletti, R., Quarteri, S., Vezzalini, G. (2006). Glass mosaic tesserae from Pompeii: an archeometrical investigation. Periodico di Mineralogia 75, 25-38.

Azzoni, C.B., DI Martino, D.C., Chiavari, C., Martini, M., Sibilia, E., Vandini, M. (2002): Electron paramagnetic resonance of mosaic glasses from the Mediterranean area. Archaeometry 44, 543 - 554.

Barahona, E. (1974): Arcillas de ladrillería de la provincia de Granada, Tesis doctoral, Universidad de Granada, 210 páginas.

Barraca de Ramos, P. (1993): Excavación arqueológica en el circuito de San Pedro (Ávila) 1989-1990, Numantia, 4, 239-256.

Barraca de Ramos, P. (1999): Ávila en la Antigüedad Tardía. García Moreno, L; Rascón Marqués, S. (eds.): Complutum y las ciudades hispanas en la Antigüedad Tardía, 181-192, Alcalá de Henares.

Barraca de Ramos, P. (2012): La ciudad de Ávila en la Antigüedad Tardía, Valladolid.

Blazquez Martínez, J. M. (1993): Mosaicos romanos de España, Madrid.

Brill, R.H. (1999): Chemical analyses of early glasses. Catalogue and tables of analyses. The Corning Museum of Glass, Corning, New York.

Brill, R.H. (2002): Chemical analyses of various glasses excavated in Greece. In: Kordas, G. (Ed.), 1st International Conference Hyalos-Vitrum-Glass.
History, technology and conservation of glass in the Hellenic world Rhodes, April 1/4 2001, Athens, pp. 167-174.

Brindley G.W., Brown G. (1984). Crystal structures of Clay Minerals and their X-Ray identification, Min. Soc. $495 \mathrm{pp}$.

Centeno Cea, I.; Quintana López, J. (2003): Ab urbe condita: sobre los orígenes y la romanización de Ávila. En: Ruíz Entrecanales, R.; Centeno Cea, I.; Quintana López, J.; Cruz Sánchez, J.; Sanchidrián Gallego, J. M. J.: Mercado grande de Ávila. Excavación arqueológica y aproximación cultural a una plaza, 40 - 89, Ávila.

Degryse, P., Schneider, J. (2008). Plyni the Elder and Sr-Nd isotopes: tracing the provenance of raw material for Roman glass production, Journal of Archaeological Science, 35, 1993 -2000.

Duran, A., Perez Rodriguez,J.L., Jimenez de Haro, M.C., Franquelo, MI.I, Robador, M.D. (2011). Analytical study of Roman and Arabic wall paintings in the Patio de Banderas of Reales Alcazares' Palace using non-destructive XRD/XRF and complementary techniques, Journal of Archaeological Science, 38, 2366 -2377.

Fabián García, J. F. (2007): "Los orígenes de la ciudad de Ávila y la época antigua. Aportaciones de la arqueología al esclarecimiento de las cuestiones históricas previas a la etapa medieval". VV.AA.: Ávila en el tiempo: Homenaje al profesor Ángel Barrios, 83-111, Ávila.

Fernández Ochoa, C., Zarzalejos Prieto, M., Burkhalter Thiébaut, C; Hevia Gómez, P., Esteban Borrajo, G. (2002): Arqueominería del Sector central de Sierra Morena. Introducción al área Sisaponense. Anejos Archivo Esp. Arqueología, XXVI, CSIC. Madrid.

Foramen (2012): Labores de limpieza del pavimento musivo, en http://villaromana.diputacionavila.es /index.php?id=40.

Foster, H., Jackson, C.M. (2010): The composition of late Romano-British colourless vessel glass: glass production and consumption. Journal of Archaeological Science 37, 3068 - 3080.

Foy, D., Picon, M., Vichy, M., Thirion-Merle, V. (2003): Caractérisation des verres de la fin de l'Antiquité en Méditerranée occidentale: l'émergence de nouveaux courants commerciaux. En: Foy, D., Nenna, M.D. (Eds.), Échanges et commerce du verre dans le monde antique, Actes du colloque de l'Association Française pour l'Archéologie du Verre, Aix-en-Provence et Marseille, June 7-9 2001, éditions Monique Mergoil, Montagnac, 41 - 85.

Hayward, K.M. (2009): Roman Quarrying and Stone Supply on the Periphery - Southern England. A 
Geological Study of First Century Funeral Monuments and Monumental Architecture. British Archaeological Report, British Series 500, Archaeopress, Oxford.

Hernando Sobrino, M. R. (2005): Epigrafía romana de Ávila, Bordeaux-Madrid.

Hunt Ortiz, M.A. (2003): Prehistoric Mining and Metallurgy in South West Iberian Peninsula. BAR, International Series 1188. Archaeopress, Oxford.

Jackson, C.M. (2005): Making colourless glass in the Roman period. Archaeometry 47 (4), 763-780.

James, L. (2006): Byzantine glass mosaic tesserae: some materials considerations. Byzantine and Modern Greek Studies 30, 29 - 47.

Mangas Manjarrés, J. (1996): Aldea y ciudad en la Antigüedad hispana, Madrid.

Mariné Isidro, M. (1984): Mosaico de la villa romana de Mancera de Arriba (Ávila), Informe inédito, Ávila.

Mariné Isidro, M. (1998): La época romana. MARINÉ ISIDRO, M. (ed.): Historia de Ávila, I Prehistoria, e Historia Antigua, 281 -338, Ávila.

Moore, D.M., Reynolds, JR. D.C. (1997): X-ray Diffraction and the identification and Analysis of clay minerals ( $2^{\text {nd }}$ ed.) Oxford Univ. Pres. New York, 378-379.

Morillo, A., Salido Dominguez, J. (2011-12). La decoración arquitectónica del campamento de la Legio VII Gemina en León, Cuadernos de Prehistoria y Arqueología, 37-38 (2), 599-624.

Neal, D.S., Cosh, S.R. (2002): Roman Mosaics of Britain. Northern Britain, vol. I. Illuminata Press with the Society of Antiquaries of London, London.

Neal, D.S., Cosh, S.R. (2009): Roman Mosaics of Britain. South-East Britain. Part 1, vol. III. The Society of Antiquaries of London, London.

Quintana López, J.; Centeno Cea, I.; Ruíz Entrecanales, R. (2003-2004): "El nacimiento de la ciudad de Ávila. Nuevos datos a partir de las cerámicas del mercedo Grande", Boletín del Seminario de Arte y Arqueología: BSAA, 69-70, 147-178.

Rodríguez Almeida, E. (2003): Ávila romana. Notas para la arqueología, la topografía y la epigrafía romanas de la ciudad y su territorio, $2^{\mathrm{a}}$ edición, Ávila.

Ruffini, A., Fiori, C., Vandini, M. (1999): Caratterizzazione chimica di vetri musivi antichi. Parte 1: metodologie di analisi e risultati. Ceramurgia 29, 285 - 298.

Schultz, L.G. (1964): Quantitative interpretation of the mineralogical composition from $X$-ray and Chemical data for Pierre Shale. U.S. Geol. Surv. Prof. Paper 391C, 1-31.

Shortland, A., Rogers, N., Eremin, K. (2007): Trace element discriminants between Egyptian and Mesopotamian Late Bronze Age glasses. Journal of Archaeological Science 34, 781-789.

Silvestri, A., Molin, G., Salviulo, G. (2005): Roman and Medieval glass from the Italian area: bulk characterisation and relationships with production technology. Archaeometry 47, 797-816.

Silvestri, A., Molin, G., Salviulo, G. (2006): Sand for Roman glass production: an experimental and philological study on source of supply Archaeometry 48, 415-432.

Silvestri, A., Molin, G., Salviulo, G. (2008): The colourless glass of Iulia Felix. Journal of Archaeological Science 35, 331-341.

Vandini, M., Fiori, C., Cametti, R. (2006): Classification and technology of Byzantine mosaic glass. Annali di Chimica 96, 587-599.

Young, J.R., Cook, S.R., Fulford, M.G., Lott, G.K. (2008): The application of microfossils in assessing the provenance of chalk used in the manufacture of Roman mosaics at Silchester. Journal of Archaeological Science 35, 2415-2422. 
